\title{
Comparación del movimiento del canino y molar superior utilizando elásticos de clase II con y sin Sliding Jig: un ensayo clínico aleatorizado
}
Comparison of the movement of the upper canine and molar using class II elastics with and without Sliding Jig: a randomized clinical trial

Comparação do movimento canino e molar superior usando elásticos classe II com e sem Sliding Jig: um ensaio clínico randomizado

\section{Luis Alejandro Sánchez Uribe ${ }^{1}$ Juan Carlos Upegui Zea² Ana María Urrea Guarín ${ }^{3}$ Ivonne Galeano Maldonado 4 Anny Vivares Builes 5}

Recibido: 07 de abril del 2019 Aprobado: 20 de junio del 2019

Publicado: 1 de julio de 2019

Cómo citar este artículo: Sánchez Uribe LA, Upegui JC, Urrea Guarín AM, Galeano Maldonado I, Vivares Builes A. Comparación del movimiento del canino y molar superior utilizando elásticos de clase II con y sin Sliding Jig: un ensayo clínico aleatorizado. Rev Nac Odontol. 2019;15(29):1-18. doi: https://doi.org/10.16925/2357-4607.2019.02.03

Artículo de investigación. https://doi.org/10.16925/2357-4607.2019.02.03

1 Facultad de Odontología, Universidad de Antioquia, Medellín, Colombia. ORCID: https://orcid.org/0000-0003-4579-5532

Facultad de Odontología, Universidad de Antioquia. Calle 64 № 52-59. Medellín, Colombia.

Correo electrónico: luis.sanchez@udea.edu.co

2 Facultad de Odontología, Universidad de Antioquia, Medellín, Colombia. ORCID: https://orcid.org/0000-0002-9618-2640

3 Facultad de Odontología, Universidad de Antioquia, Medellín, Colombia. ORCID: https://orcid.org/0000-0002-8048-7650

4 Facultad de Odontología, Universidad de Antioquia, Medellín, Colombia. ORCID: https://orcid.org/0000-0002-5805-0962

5 Facultad de Odontología, Universidad de Antioquia, Medellín, Colombia. ORCID: https://orcid.org/0000-0002-8631-4910 


\section{Resumen}

Introducción: para lograr relaciones caninas de clase I, se utilizan elásticos intermaxilares de clase II. El Sliding Jig es un aditamento que ejerce fuerza distal directa sobre el molar, potenciando el vector horizontal del elástico intermaxilar. Objetivo: Determinar y comparar, en un periodo de tres meses, el tipo y la cantidad de movimiento sagital y vertical del molar superior y del canino utilizando elásticos de clase II con y sin Sliding Jig. Métodos: se realizó un ensayo clínico aleatorizado, no controlado sin cegamiento; se compararon 15 hemiarcadas tratadas con Sliding Jig, con 14 hemiarcadas tratadas solo con elásticos clase II. Los movimientos fueron evaluados por un investigador calibrado (ICC:>0,8), en una Tomografía Computarizada Cone Beam (CBCT) del maxilar superior inicial ( $\mathrm{T} 1$ ) y una $\mathrm{CBCT}$ final (T2) a los tres meses de instalada la mecánica.

Resultados: las variables fueron resumidas en promedios y desviaciones estándar con un intervalo de confianza del $95 \%$; para la comparación de los resultados entre T1 y T2 se utilizó la prueba t-Student. Se encontró mayor distalización del molar superior y menor movimiento vertical del canino superior, en el lado de Sliding Jig comparado con el lado de solo elásticos, mostrando diferencias estadísticamente significativas $(p<0,05)$. No se presentaron cambios significativos en los movimientos sagital y de inclinación del canino, ni en la inclinación del molar entre las dos mecánicas.

Conclusiones: el Sliding Jig fue más eficiente que los elásticos de clase II solos, en la distalización de los molares superiores y en el control de la extrusión del canino.

Palabras clave: ortodoncia, maloclusión Angle clase II, técnicas de movimiento dental, ortodoncia correctiva.

\section{Abstract}

Introduction: For the achievement of a cuspid Class I relationship, intermaxillary class II elastics have been used. They generate retraction in the upper arch and protraction on the lower arch. The Sliding Jig is a direct attachment, which exerts distal force on the molar, enhancing the horizontal vector of the intermaxillary elastic.

Objective: To determin in a period of three months, the type and amount of movement of the upper canine and molar in the sagittal and vertical plane using class II elastics with and without Sliding Jig.

Method: An uncontrolled randomized clinical trial was conducted to compare 15 hemiarches treated with Sliding Jig and 14 treated with class II elastics alone. The movements of each side were evaluated by a calibrated evaluator, using an initial Cone Beam Computed Tomography (CBCT) (T1) and a final CBCT (T2), taking three months after the mechanism was installed.

Results: Variables were summarized using mean and standard deviations with a $95 \%$ confidence interval. For $\mathrm{T} 1$ and $\mathrm{T} 2$ comparison, a $t$-Student test was used. The upper molar distalization was greater and the vertical movement of the upper canine was lesser when using the Sliding Jig than when using only the elastics, with statistically significant differences $(p<0,05)$; non-significant changes in sagittal and vertical movements of the canine or molar inclination were found.

Conclusions: The Sliding Jig was more efficient than class II elastics alone, on the distalization of upper molars and the control of the canine extrusion.

Keywords: Orthodontic Appliances, Maloclussion Angle Class II, Tooth Movement Techniques, Corrective Orthodontics. 


\section{Resumo}

Introdução: para alcançar relações caninas da classe I, são utilizados elásticos intermaxilares da classe II. O Sliding Jig é um acessório que exerce força distal direta no molar, aprimorando o vetor horizontal do elástico intermaxilar. Objetivo: Determinar e comparar, em um período de três meses, o tipo e a quantidade de movimento sagital e vertical do molar superior e do canino usando elásticos classe II com e sem gabarito deslizante.

Métodos: foi realizado um ensaio clínico randomizado, não controlado, sem cegar; 15 hemiarquias tratadas com Sliding Jig foram comparadas com 14 hemiarquias tratadas apenas com elásticos de classe II. Os movimentos foram avaliados por um investigador calibrado (ICC:>0,8), em uma tomografia computadorizada de feixe cônico (TCFC) da mandíbula superior inicial (T1) e uma TCFC final (T2) três meses após a instalação da mecânica.

Resultados: as variáveis foram resumidas em média e desvio padrão com intervalo de confiança de 95\%; Para a comparação dos resultados entre T1 e T2, foi utilizado o teste t de Student. Maior distalização do molar superior e menor movimento vertical do canino superior foram encontradas no lado do Sliding Jig. comparado ao lado elástico, mostrando diferenças estatisticamente significantes $(p<0,05)$. Não houve alterações significativas nos movimentos sagital e de inclinação do canino, nem na inclinação molar entre as duas mecânicas.

Conclusões: o Sliding Jig foi mais eficiente que os elásticos classe Il isoladamente, na distalização dos molares superiores e no controle da extrusão do canino.

Palavras-chave: ortodontia, má oclusão de ângulo classe II, técnicas de movimentação dentária, ortodontia corretiva.

\section{Introducción}

La maloclusión clase II puede deberse a una combinación de alteraciones esqueléticas y dentarias originadas por factores genéticos, ambientales y funcionales $(1,2)$; entre los métodos utilizados para su corrección está la distalización de molares superiores (3). Actualmente se implementan técnicas intrabucales que brindan mayor comodidad y aceptación por los pacientes, como son los elásticos intermaxilares, el uso de coil spring, sistemas pendulares, el Jones Jig y el Sliding Jig, entre otros $(4,6)$. Se ha reportado que la fuerza necesaria para el movimiento distal del segmento posterior y de los caninos en cuerpo es entre 120 a 200 gr (7).

Entre los más utilizados están los elásticos intermaxilares los cuales presentan buenas características mecánicas, como adecuada resistencia tensil, buen módulo de elasticidad, biocompatibilidad y bajo costo (8); elaborados en poliuretano se alteran con la temperatura, el $\mathrm{pH}$ intraoral y el tiempo, y presentan un deterioro aproximado de $32,6 \%$ del nivel de su fuerza a las 24 horas $(9,10)$. Los elásticos de clase II se utilizan desde el canino o incisivo lateral superior hasta el primer o segundo molar inferior; debido a que la fuerza no es paralela al plano oclusal producen vectores en sentido horizontal, transversal y vertical, generando fuerzas extrusivas en los sitios donde se 
anclan (11). El efecto distalizador de los elásticos de clase II recae solo en el canino o el incisivo lateral superior sin que el molar superior reciba directamente una fuerza distalizadora, lo que ocasiona que ésta mecánica sea poco eficiente en la corrección de la relación de clase II molar (12).

Buscando minimizar los efectos indeseados de los elásticos intermaxilares se desarrolló el Sliding Jig, descrito por primera vez en 1966 por Tweed, se utilizó para distalizar los dientes superiores apoyado en un anclaje dental inferior (13); consiste en un gancho deslizante elaborado con un segmento de alambre de acero inoxidable que se desliza sobre el arco principal por la acción de un elástico intermaxilar que va anclado desde un gancho en forma de $\mathrm{J}$ del Sliding Jig ubicado entre el lateral y canino superior con un vector de clase II hasta la arcada opuesta, el gancho deslizante recibe el efecto de distalización y transmite una fuerza distal al molar, haciendo más eficiente la fuerza generada por el elástico y al estar el gancho en J ubicado por debajo del plano oclusal superior disminuye los efectos colaterales verticales del uso de los elásticos $(14,15)$. A través del tiempo, varios autores le han realizado modificaciones al diseño original del Sliding Jig, utilizándolo tanto para la corrección de las relaciones oclusales de clase II, como las de clase III $(14,16,17)$. Para los casos de maloclusión clase II subdivisión o clase II unilaterales, en la literatura se ha reportado el uso de otro tipo de auxiliares como son el Forsus (18) y los minitornillos (19).

Las tomografías Cone Beam han demostrado ser una gran herramienta en el diagnóstico y en la evaluación de los diferentes tratamientos ortodóncicos. Su uso es cada vez más popular y el detalle y la facilidad de medición que ofrecen, la convierten en un instrumento útil y confiable con fines investigativos $(20,21)$.

Hasta el momento es escasa la evidencia científica que reporte los efectos dentoalveolares del Sliding Jig en la corrección de la relación dental de clase II; el propósito de esta investigación fue establecer y comparar los tipos de movimientos (traslación/inclinación) en el plano sagital y vertical del canino y el molar superior obtenidos con imágenes tomográficas Cone Beam con el uso de elásticos de clase II con y sin Sliding Jig durante 3 meses de tratamiento en pacientes en los cuales estuviese indicado este tipo de mecánica. 


\section{Materiales y métodos}

\section{Diseño del estudio}

Este ensayo clínico aleatorizado, no controlado sin cegamiento, se registró en el Instituto Nacional de Salud de los Estados Unidos (ClinicalTrials.gov) con el numero NTC01654419. Se compararon 30 unidades de análisis; la unidad de análisis fue definida como una hemiarcada y se les instaló el Sliding Jig o los elásticos solos. Algunos pacientes aportaron las dos hemiarcadas, y en cada una de ellas se colocó una u otra mecánica, igualmente hubo pacientes que aportaron solo una hemiarcada, la cual fue aleatorizada para cualquiera de las dos mecánicas. Se trataron 15 unidades de análisis con Sliding Jig acompañado de elásticos y otras 15 fueron tratadas solo con elásticos; una de las unidades de análisis del grupo tratado solo con elásticos fue excluida debido a errores en la posición cefálica durante la toma de la imagen tomográfica final, quedando conformado este grupo con 14 unidades.

La muestra fue seleccionada a conveniencia de la población de pacientes en tratamiento de ortodoncia en la Facultad de Odontología de la Universidad de Antioquia y de la consulta particular de los investigadores. Los siguientes fueron los criterios de inclusión: pacientes de 13 a 40 años con tratamiento de ortodoncia que hayan terminado la fase de alineación y nivelación; sin sensibilidad vertical, con brackets con prescripción de Roth con slot 0,018×0,025"; relación canina y molar clase II unilateral o bilateral con discrepancias de 2 a 4 mm; caninos y molares superiores con formación radicular completa sin historia de trauma, patología periodontal, pulpar o periradicular; dentición permanente con los segundos molares erupcionados; terceros molares ausentes clínicamente; óptimas condiciones periodontales y de higiene oral.

\section{Procedimiento clínico}

El diseño que se eligió para confeccionar el Sliding Jig fue el propuesto por Lucato et al. en 2003 (6), utilizando un calibre 0,017×0.025" de acero inoxidable, fue construido por un sólo investigador y su diseño fue verificado por el investigador principal (figura 1), se le instaló a cada paciente en la arcada superior un arco de acero 0,016x0,016" con ligaduras metálicas individuales y en la arcada inferior un arco de acero 0,016x0,022" consolidado con ligadura metálica continua de primer molar derecho a primer molar izquierdo; a continuación se cementó un segmento de alambre de acero 0,016x0,022" de $2 \mathrm{~mm}$ de largo en la cúspide del canino superior a distalizar, como marcador para realizar las mediciones en la tomografía. 


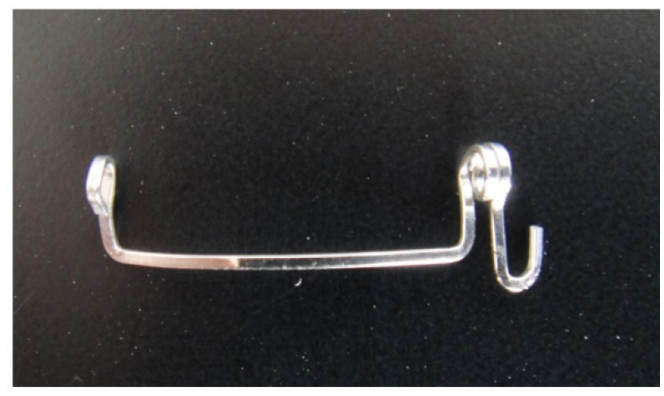

Figura 1. Diseño Sliding Jig.

Fuente: elaboración propia

Los pacientes fueron aleatorizados para las dos mecánicas mediante un muestreo aleatorio simple. El grupo al que se le instaló el Sliding Jig estuvo conformado por 11 mujeres y 4 hombres, se les indicó la utilización de los elásticos desde el gancho en $J$ hasta el hook del tubo del primer molar inferior; el grupo con elásticos solos estuvo conformado por 10 mujeres y 4 hombres, se les indicó la utilización de los elásticos desde el hook del bracket del canino superior hasta el hook del tubo del primer molar inferior (figura 2). A los pacientes de ambos grupos se les explicó que debían usar elásticos con una fuerza de 6 onzas (170 gramos) y un diámetro de 3/16 (4.76 mm) 24 horas al día y cambiarlos diariamente, registrando las horas de uso diario en una planilla de seguimiento que le fue entregada a cada individuo. Los pacientes fueron evaluados cada 15 días para motivarlos en el uso de los elásticos y verificar que el proceso se estuviera llevando a cabo de forma adecuada.
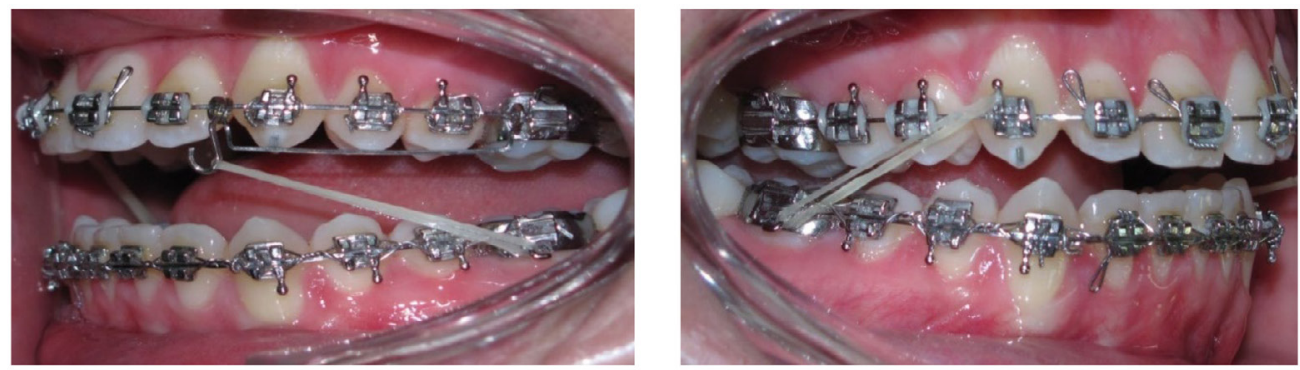

Figura 2. Elásticos con (a) y sin (b) Sliding Jig.

Fuente: elaboración propia 


\section{Medición}

Cada uno de los integrantes de la muestra se documentó con una Tomografía Computarizada Cone Beam (CBCT) del maxilar superior inicial (T1) y una CBCT final (T2) a los tres meses de instalada la mecánica, se tomaron con la boca abierta y con el plano Frankfort perpendicular al piso. Las CBCT fueron tomadas con un equipo tomográfico Newton $3 \mathrm{G}^{\oplus}$. Los factores de kilovoltaje (Kvp) y miliamperaje $(\mathrm{mA})$ fueron calculados automáticamente por el equipo de acuerdo a la densidad de la estructura corporal de cada paciente. A partir de las imágenes axiales se realizó un corte panorámico con un corredor focal de $25 \mathrm{~mm}$; la imagen axial utilizada para este corte se seleccionó desde la imagen exploradora en el corte correspondiente a la unión cementoamélica en distal del segundo molar superior. Los puntos para el trazo de corte de la vista panorámica se hicieron siguiendo la mitad de las coronas en los cortes axiales, correspondiente a las cámaras pulpares. Los puntos y planos de referencia fueron construidos sobre el corte panorámico por el mismo operador con una concordancia intraobservador mediante el coeficiente de correlación intraclase con valores que oscilaron entre $(0,89$ y 0,98$)$, con un promedio de la diferencia entre los dos trazos de $0,46 \pm 0,51 \mathrm{~mm}$. Las mediciones lineales y angulares fueron realizadas por el mismo operador, desconociendo el tipo de mecánica que se había aplicado en cada unidad de análisis, mediante el software NNT Station del modelo Newton 3G (figura 3).

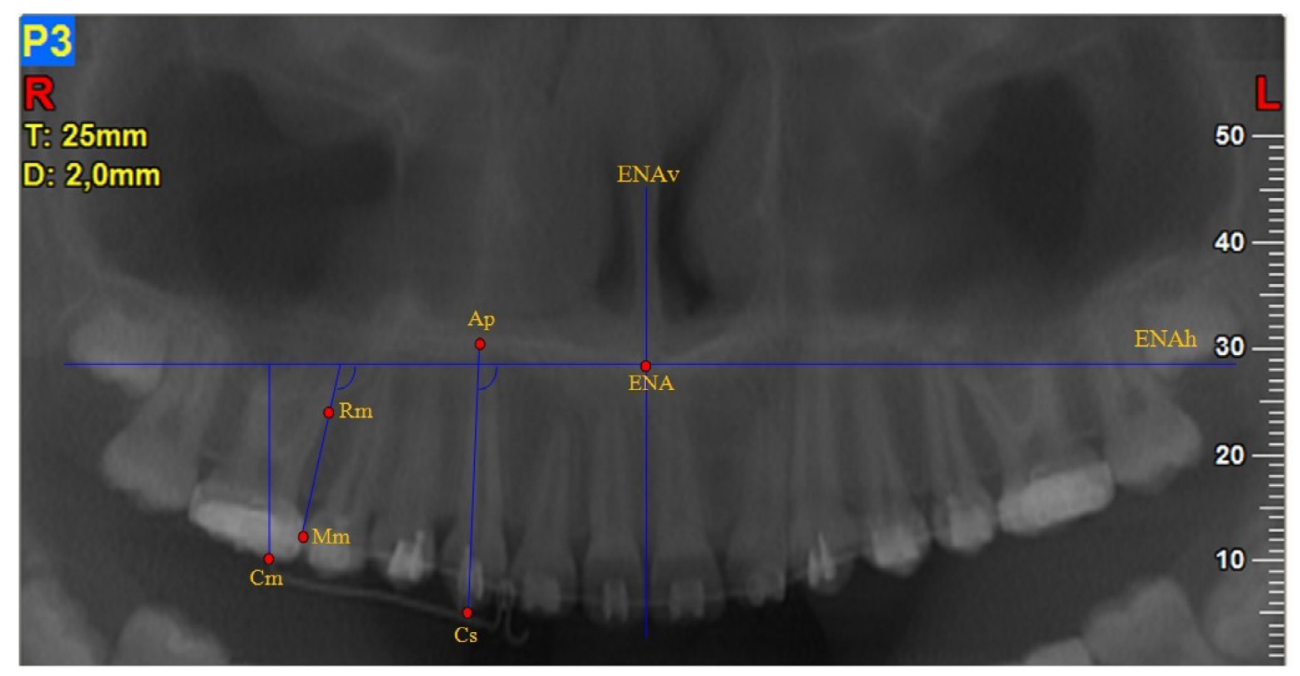

Figura 3. Variables tomográficas.

Fuente: elaboración propia 
Tabla 1. Puntos, planos y ángulos de referencia usados para la medición de cambios dentolaveolares.

\begin{tabular}{ccc}
\hline Puntos de referencia & Planos construidos & Ángulos construidos \\
\hline $\begin{array}{c}\text { ENA: estructura de la espina } \\
\text { nasal anterior. }\end{array}$ & $\begin{array}{c}\text { ENAv: línea vertical que } \\
\text { pasa por ENA. }\end{array}$ & $\begin{array}{c}\text { Ángulo ENAh - Eje del canino } \\
\text { superior: se midió el ángulo } \\
\text { inferior interno. }\end{array}$ \\
\hline $\begin{array}{c}\text { Cs: parte más incisal de la } \\
\text { señal metálica del canino } \\
\text { superior. }\end{array}$ & $\begin{array}{c}\text { ENAh: línea horizontal } \\
\text { que pasa por ENA } \\
\text { perpendicular a ENAv. }\end{array}$ & $\begin{array}{c}\text { Ángulo ENAh - Eje del molar } \\
\text { superior: se midió el ángulo } \\
\text { inferior interno. }\end{array}$ \\
\hline $\begin{array}{c}\text { Ap: punto más apical de la } \\
\text { raíz del canino superior. }\end{array}$ & $\begin{array}{c}\text { Eje del canino superior: } \\
\text { línea que une los puntos } \\
\text { Ap y Cs. }\end{array}$ & \\
\hline $\begin{array}{c}\text { Mm: punto más convexo de la } \\
\text { superficie mesial del primer } \\
\text { molar superior }\end{array}$ & $\begin{array}{c}\text { Eje del molar superior: } \\
\text { línea que una los puntos }\end{array}$ & \\
\hline $\begin{array}{c}\text { Rm: punto más mesial de } \\
\text { la raíz mesiovestibular del } \\
\text { primer molar superior. }\end{array}$ & & \\
\hline $\begin{array}{c}\text { Cm: cúspide mesial del } \\
\text { primer molar superior }\end{array}$ & & \\
\hline
\end{tabular}

Fuente: elaboración propia

Los cambios sagitales y verticales se obtuvieron con las diferencias entre las medidas en T2-T1.

Los cambios sagitales del canino se establecieron midiendo la distancia en milímetros desde Cs hasta ENAv con una línea perpendicular al plano ENAv y en el molar midiendo la distancia en milímetros desde Mm hasta ENAv con una línea perpendicular al plano ENAv.

Los cambios en la inclinación del canino superior se determinaron midiendo el ángulo inferior interno formado por ENAh y el eje del canino superior y la inclinación del molar superior se determinó midiendo el ángulo inferior interno formado por ENAh y el eje del molar superior.

Los cambios verticales del canino se establecieron midiendo la distancia en milímetros desde Cs hasta el plano ENAh con una línea perpendicular a ENAh y los del primer molar superior se determinaron midiendo la distancia en milímetros desde Cm hasta el plano ENAh con una línea perpendicular a ENAh. 


\section{Aspectos éticos}

Los pacientes y acudientes recibieron la información sobre los objetivos del estudio y se solicitó su participación y la firma del consentimiento informado en presencia de testigos; cumpliendo con los requerimientos éticos de la investigación en salud según la Resolución 008430 del Ministerio de Salud y Protección Social de Colombia, las recomendaciones de la declaración de Helsinki y además, con el aval del Comité de Ética Institucional (CIFO) de la Universidad de Antioquia en la Resolución 001-2011.

\section{Análisis estadístico}

La digitalización de la información obtenida se realizó mediante una base de datos en el programa Excel para Microsoft. Para el análisis estadístico de la información se utilizó el programa SPSS versión 19 (SPSS Inc., Chicago IL). Todas las variables tomográficas presentaron distribución normal mediante la prueba de Shapiro Wilk, a excepción de la variable MV (molar vertical). Por lo que se presentan en promedios, desviaciones estándar y con un intervalo de confianza del 95\%. Para la comparación de los resultados entre T1 y T2 se utilizó la prueba $\boldsymbol{t}$-Student para muestras pareadas. Siempre se asumió un nivel de significancia del $5 \%$ (donde los valores de $p<0,05$ fueron considerados estadísticamente significativos).

\section{Resultados}

Se evaluaron 15 unidades de análisis en el grupo de Sliding Jig con un promedio de

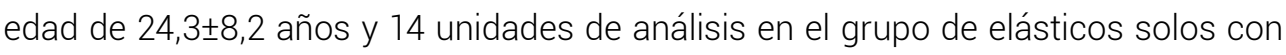
un promedio de edad de 22,9 97,3 años. El promedio de horas de uso de los elásticos fue mayor en el grupo con Sliding Jig (1.824 horas) comparado con el grupo de elásticos solos (1.788 horas), esto puede estar asociado a la incomodidad que se produce al utilizar el Sliding Jig sin el uso de elásticos ya que son estos últimos los que lo estabilizan en el arco; sin embargo, las diferencias no fueron estadísticamente significativas. Las condiciones basales para ambos grupos fueron similares (tabla 2). 
Tabla 2. Descripción de las condiciones basales de las medidas tomográficas entre los dos grupos.

\begin{tabular}{|c|c|c|c|c|c|c|}
\hline \multirow{2}{*}{ Medida } & \multirow{2}{*}{ grupo } & \multirow{2}{*}{$n$} & \multirow{2}{*}{ Media $\pm D E$} & \multicolumn{2}{|l|}{ IC $95 \%$} & \multirow{2}{*}{ Valor p } \\
\hline & & & & Lim. Inf. & Lim. Sup & \\
\hline \multirow{2}{*}{ CST1 } & Sliding Jig & 15 & $16,2 \pm 1,5$ & 15,4 & 17,1 & \multirow{2}{*}{0,714} \\
\hline & Elásticos & 14 & $16,4 \pm 1,1$ & 15,8 & 17,1 & \\
\hline \multirow{2}{*}{ CVT1 } & Sliding Jig & 15 & $24,5 \pm 3,6$ & 22,6 & 26,5 & \multirow{2}{*}{0,244} \\
\hline & Elásticos & 14 & $26,0 \pm 3,1$ & 24,2 & 27,8 & \\
\hline \multirow{2}{*}{$\mathrm{ClT} 1$} & Sliding Jig & 15 & $84,7 \pm 4,5$ & 82,2 & 87,2 & \multirow{2}{*}{0,572} \\
\hline & Elásticos & 14 & $83,4 \pm 7,1$ & 79,3 & 87,5 & \\
\hline \multirow{2}{*}{ MST1 } & Sliding Jig & 15 & $33,2 \pm 2,3$ & 31,9 & 34,4 & \multirow{2}{*}{0,309} \\
\hline & Elásticos & 14 & $34,1 \pm 2,6$ & 32,6 & 35,6 & \\
\hline \multirow{2}{*}{ MVT1 } & Sliding Jig & 15 & $22,2 \pm 2,6$ & 20,7 & 23,6 & \multirow{2}{*}{0,307} \\
\hline & Elásticos & 14 & $23,2 \pm 2,9$ & 21,6 & 24,9 & \\
\hline \multirow{2}{*}{ MIT1 } & Sliding Jig & 15 & $86,6 \pm 8,9$ & 81,6 & 91,5 & \multirow{2}{*}{0,644} \\
\hline & Elásticos & 14 & $87,9 \pm 6,1$ & 84,4 & 91,4 & \\
\hline
\end{tabular}

*t- test no pareada $p<0,05$

Fuente: elaboración propia

Al comparar las variables del grupo con Sliding Jig antes y después en el análisis tomográfico se presentaron cambios significativos en todas las variables; el mayor movimiento fue la inclinación del molar y el que menos se expresó fue el movimiento vertical del canino. En el grupo con Sliding Jig el promedio de distalización del canino fue de 1,02 $\pm 0,55 \mathrm{~mm}$; y en el grupo con elásticos solos el promedio de distalización fue de 1,06 $\pm 0,54 \mathrm{~mm}$.

Con relación al movimiento vertical del canino, el grupo con Sliding Jig presentó en promedio una extrusión del canino de 0,30 $\pm 0,48 \mathrm{~mm}$, mientras que el grupo de

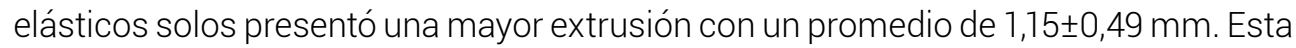
diferencia fue estadísticamente significativa. Cuando se evaluó el movimiento vertical del molar superior, el grupo con Sliding Jig tuvo un promedio de movimiento extrusivo

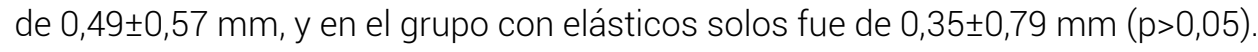

Se encontró que el promedio de la inclinación del molar en el grupo con Sliding Jig fue de 2,17 $\pm 1,86^{\circ}$ y el promedio de inclinación en el grupo con elásticos solos fue de $1,91 \pm 1,70^{\circ}$, aunque no se presentaron diferencias significativas en la inclinación de los molares con las dos mecánicas. Las comparaciones de los movimientos en molares y caninos se observan en la tabla 3. 
Tabla 3. Diferencias entre T2 y T1 en el grupo con Sliding Jig.

\begin{tabular}{ccccc}
\hline \multirow{2}{*}{ Variables } & Media \pm DE & \multicolumn{3}{c}{ Intervalo de confianza 95 \% } \\
\cline { 3 - 5 } & & Límite inferior & Limite superior & Valor P \\
\hline CST2 - CST1 & $1,02 \pm 0,55$ & 0,72 & 1,32 & $0,000^{*}$ \\
\hline CVT2 - CVT1 & $0,30 \pm 0,48$ & 0,04 & 0,56 & $0,029^{*}$ \\
\hline CIT2 - CIT1 & $1,83 \pm 1,21$ & 1,16 & 2,50 & $0,000^{*}$ \\
\hline MST2 - MST1 & $1,25 \pm 0,70$ & 0,86 & 1,63 & $0,000^{*}$ \\
\hline MVT2 - MVT1 & $0,49 \pm 0,57$ & 0,17 & 0,80 & $0,005^{*}$ \\
\hline MIT2 - MIT1 & $2,17 \pm 1,86$ & 1,14 & 3,21 & $0,000^{*}$ \\
\hline
\end{tabular}

T1: Tomografía inicial, T2: Tomografía final. CS: Distancia canino sagital.Cl: Inclinación del canino.

CV: Distancia del canino vertical. MS: Distancia del molar sagital. MV: Distancia molar vertical.

MI: Inclinación del molar.

*t-Student $p<0,05$

Fuente: elaboración propia

Cuando se compararon las variables del grupo de elásticos solos se encontraron diferencias estadísticamente significativas para cinco de las variables; el movimiento vertical del molar fue el que menos se expresó y fue en esta variable donde no se encontraron diferencias significativas. El movimiento que más se expresó fue la inclinación distal del canino (tabla 4).

Tabla 4. Diferencias entre T2 y T1 en el grupo con elásticos solos.

\begin{tabular}{ccccc}
\hline \multirow{2}{*}{ Variables } & $\begin{array}{c}\text { Media } \pm \mathbf{D E} \\
\text { Límite inferior }\end{array}$ & \multicolumn{2}{c}{ Intervalo de confianza 95\% } & \multirow{2}{*}{ Valor $\mathbf{P}$} \\
\cline { 3 - 4 } & $\mathbf{2}$ Límite superior & & \\
\hline CST2 - CST1 & $1,06 \pm 0,54$ & 0,75 & 1,37 & $0,000^{*}$ \\
\hline CVT2 - CVT1 & $1,15 \pm 0,49$ & 0,86 & 1,43 & $0,000^{*}$ \\
\hline CIT2 - CIT1 & $2,20 \pm 1,96$ & 1,07 & 3,34 & $0,001^{*}$ \\
\hline MST2 - MST1 & $0,40 \pm 0,32$ & 0,21 & 0,59 & $0,000^{*}$ \\
\hline MVT2 - MVT1 & $0,35 \pm 0,79$ & 0,10 & 0,81 & $0,116^{*}$ \\
\hline MIT2 - MIT1 & $1,91 \pm 1,70$ & 0,92 & 2,90 & $0,001^{*}$ \\
\hline
\end{tabular}

CST1-CST2: Diferencia del movimiento sagital del canino.

CVT1-CVT2: Diferencia del movimiento vertical del canino.

CIT1-CIT2: Diferencia de la inclinación del canino.

MST1-MST2: Diferencia del movimiento sagital del molar.

MVT1-MVT2: Diferencia del movimiento vertical del molar.

MIT1-MIT2: Diferencia de la inclinación del molar.

*t-test pareada $p<0,05$

Fuente: elaboración propia 
En las medidas tomográficas se pudo observar que las unidades de análisis del grupo con Sliding Jig presentaron una mayor distalización del molar superior y menor movimiento vertical del canino superior comparado con las unidades de análisis del grupo de elásticos solos con diferencias estadísticamente significativas $(p<0,05)$. No se observaron cambios significativos en los movimientos sagitales y de inclinación del canino, ni en la inclinación del molar cuando se compararon los dos grupos (tabla 5).

Tabla 5. Comparación del movimiento del canino y molar, superior con Sliding Jig y elásticos solos.

\begin{tabular}{|c|c|c|c|c|}
\hline Variable & Mecánica & $\mathrm{N}$ & Media \pm D E & Valor $\mathrm{P}$ \\
\hline \multirow{2}{*}{ CSDIF } & Sliding Jig & 15 & $1,02 \pm 0,54$ & \multirow{2}{*}{0,828} \\
\hline & Elásticos & 14 & $1,06 \pm 0,54$ & \\
\hline \multirow{2}{*}{ CVDIF } & Sliding Jig & 14 & $0,34 \pm 0,43$ & \multirow{2}{*}{$0,000^{*}$} \\
\hline & Elásticos & 15 & $1,16 \pm 0,49$ & \\
\hline \multirow{2}{*}{ CIDIF } & Sliding Jig & 14 & $1,71 \pm 1,17$ & \multirow{2}{*}{0,427} \\
\hline & Elásticos & 15 & $2,20 \pm 1,96$ & \\
\hline \multirow{2}{*}{ MSDIF } & Sliding Jig & 15 & $1,24 \pm 0,69$ & \multirow{2}{*}{$0,003^{*}$} \\
\hline & Elásticos & 14 & $0,50 \pm 0,47$ & \\
\hline \multirow[t]{2}{*}{ MVDIF } & Sliding Jig & 14 & $0,57 \pm 0,48$ & \multirow[t]{2}{*}{0,893} \\
\hline & Elásticos & 15 & $0,60 \pm 0,61$ & \\
\hline \multirow{2}{*}{ MIDIF } & Sliding Jig & 14 & $1,807 \pm 1,25$ & \multirow{2}{*}{0,813} \\
\hline & Elásticos & 15 & $1,943 \pm 1,71$ & \\
\hline
\end{tabular}

t-test $p<0,05$

CSDIF: Diferencia del movimiento sagital del canino con Sliding Jig y elásticos. CVDIF: Diferencia del movimiento vertical del canino con Sliding Jig y elásticos. CIDIF: Diferencia de la inclinación del canino con Sliding Jig y elásticos. MSDIF: Diferencia del movimiento sagital del molar con Sliding Jig y elásticos. MVDIF: Diferencia del movimiento vertical del molar con Sliding Jig y elásticos.

Fuente: elaboración propia

\section{Discusión}

La distalización de los molares superiores es a menudo un reto en el tratamiento ortodóncico ya que frecuentemente ocurren efectos indeseables con las biomecánicas utilizadas, es por esto que el movimiento durante la distalización requiere una evaluación y control en los planos del espacio. La utilización de tomografías para la valoración de movimientos dentales ha aumentado en los últimos años (22), ya que la precisión en las mediciones y en los detalles es mucho mejor que con radiografías convencionales o modelos. 
El Sliding Jig es un gancho deslizante asociado a elásticos de clase II indicado para el movimiento de los dientes superiores en sentido distal (13). Los resultados obtenidos en las medidas tomográficas indican que las unidades de análisis que recibieron tratamiento con el Sliding Jig presentaron una mayor distalización del molar superior y menor movimiento vertical del canino superior comparado con las unidades de análisis que recibieron tratamiento con elásticos solos.

El análisis de los movimientos de los molares y caninos, nos indican el desempeño de las mecánicas ortodoncicas. Con relación al movimiento distal del canino superior en el plano sagital, en ambos grupos, no se obtuvieron diferencias, lo que demuestra que ambas mecánicas produjeron un movimiento similar del canino hacia distal. En el caso del Sliding Jig aunque no se ejerce una fuerza directa sobre el canino, éste y los premolares se mueven espontáneamente hacia distal cuando se distaliza el molar debido, probablemente, a la acción de los grupos de fibras gingivales que presentan una interconexión con los dientes vecinos, efecto reportado por otros autores en estudios de distalización (23).

Uno de los efectos indeseables de los elásticos de clase II es la extrusión de los dientes donde se anclan. En la presente investigación se encontró menor extrusión del canino superior en el grupo de Sliding Jig. Este hallazgo puede explicarse por el hecho de que el elástico solo se ancla directamente en el canino y presenta un mayor componente vertical comparado con el Sliding Jig el cual, debido a su diseño, disminuye el vector vertical al anclar el elástico en el gancho que se encuentra por debajo del plano oclusal superior. Efectos verticales similares con elásticos de clase II fueron encontrados por Philippe en 1995 (11).

Aunque la diferencia en la inclinación del canino superior entre los dos grupos, no fue estadísticamente significativa, la mayor aparente inclinación en el grupo con elásticos solos se podría explicar debido a que con los elásticos la fuerza se aplica directamente sobre el canino produciendo inicialmente un movimiento coronal que lo inclina a distal, sin alteración de la posición radicular.

La presente investigación encontró que en el grupo con Sliding Jig el promedio de distalización del molar fue mayor que en el grupo de elásticos solos. Estos resultados se podrían relacionar con el hecho de que la fuerza que producen los elásticos no recae directamente sobre el molar por lo que no se genera movimiento significativo en el grupo con elásticos solos, mientras que el diseño del Sliding Jig permite un contacto directo entre el loop distal del gancho y el tubo del primer molar ejerciendo una fuerza distalizadora directa. Estos hallazgos son similares a los Patel et al. en el 2009 (24), quienes encontraron que el apoyo directo sobre el molar, tienen un efecto distalizador, tal y como se observa con aditamentos como el péndulo y con el Jones Jig. 
Por otro lado, al analizar los efectos dentoalveolares del molar superior en el plano vertical, los valores bajos en estas medidas se explican porque ninguna de las dos mecánicas genera vectores de fuerza verticales que recaen directamente sobre el molar. Los resultados también revelan que no se presentaron diferencias significativas en la inclinación de los molares con las dos mecánicas, aunque en el grupo con Sliding Jig fue mayor debido, probablemente, a que la fuerza se dirige directamente sobre el molar. La inclinación del molar también se ha reportado por otros autores con diferentes tipos de aparatología $(23,24)$.

La evidencia científica relacionada con el Sliding Jig es escasa; el primer reporte que se conoce de esta mecánica fue el realizado por Tweed en 1966 (13), posteriormente Richard Adams, en 1985 (25), patentó el diseño de un gancho deslizante distalizador, ambos reportes solo describen el diseño más no evalúan ni comparan su efectividad. Quirós, en 1993 (26), reportó la utilización de un Sliding Jig con una mecánica modificada al utilizar resortes abiertos de Níquel-Titanio, el autor hace una descripción de la mecánica más no reporta datos que demuestren su efectividad comparándola con la mecánica convencional propuesta por Tweed. Burbano et al., en 2007 (15), realizaron un estudio en 20 pacientes donde reportaron una modificación del diseño original del Sliding Jig llamado gancho deslizante de protracción para la corrección de la clase II división 2 y evaluaron la efectividad de este gancho de protracción comparado con los elásticos de clase II, encontrando mayor velocidad de la corrección de la clase II con el gancho de protracción; sin embargo este estudio no es comparable con el estudio presente ya que evaluó clínicamente la protracción del canino inferior y no midió los efectos dentoalveolares producidos por estas dos mecánicas.

Alves et al. en 2006 (14), reportaron un caso con maloclusión clase III tratado con Sliding Jig obteniendo resultados favorables, sin embargo no muestran información específica acerca del tratamiento de la clase II.

Existen artículos que reportan la utilización de anclaje esquelético tanto para la corrección de la maloclusión de clase III (17) como para la clase II (19), sin embargo, debido al tipo de maloclusión tratada y al anclaje utilizado en estos estudios no es posible realizar un análisis comparativo.

La mayoría de los estudios para corrección de la clase ll sin extracciones hacen referencia a mecánicas distalizadoras tipo péndulo, Jones-Jig, First-Class, Herbst, magnetos, Distal-jet $(16,27,28)$, entre otros, los cuales producen movimientos significativamente mayores que los producidos por el Sliding Jig y los elásticos de clase II, por tal razón no es posible hacer una comparación de los resultados de este estudio con dichas mecánicas. Otras opciones terapéuticas para el manejo de la clase 
II incluyen el uso de propulsores mandibulares tipo Forsus (18). Los problemas de anclaje que se pueden presentar con el uso de estos aditamentos se pueden contrarrestar con el uso de un Sliding Jig soportado a un minitornillo (29).

El Sliding Jig inicialmente produce una distalización del molar sin observarse en algunos casos un movimiento significativo del canino y los premolares, lo que hace necesario que, en una fase posterior, se deban distalizar estos dientes e implica un tiempo adicional que aumenta el tiempo total de tratamiento. Esto puede ser una limitación de esta mecánica.

La evaluación de la metodología utilizada en este estudio permitió determinar que con el análisis tomográfico es posible evaluar el movimiento dental con un mínimo margen de error (22), sin embargo, existen factores que pueden alterar los resultados como son: la falta de estandarización de las imágenes debido a variaciones mínimas en la posición de la cabeza, movimientos del paciente durante la toma, variaciones en el corte axial, reconstrucción de la imagen panorámica y la calibración del operador; puesto que las medidas son de rango pequeño y cualquier alteración puede producir sesgo en los resultados.

\section{Conclusiones}

Durante la fase de tratamiento el Sliding Jig fue más eficiente que los elásticos de clase II en la distalización de los molares superiores y en el control de la extrusión del canino.

Se produjo mayor extrusión e inclinación del canino con el uso de elásticos solos, mientras que hubo más inclinación del molar en el grupo de Sliding Jig.

El Sliding Jig es una mecánica simple de bajo costo que puede usarse para desplazar dientes uni o bilateralmente, pero los resultados de la mecánica están limitados a la colaboración del paciente.

\section{Limitaciones del estudio}

La utilización de un alambre de acero 0,016×0,016" como arco principal pudo alterar el control rotacional y transversal, pero se utilizó con el fin de disminuir la fricción y favorecer el movimiento. Adicionalmente, algunos de los pacientes, no presentaban los segundos molares involucrados en el sistema, debido a que el objetivo del Sliding Jig era evaluar el movimiento distal del primer molar específicamente. 
Algunas de las variaciones encontradas en el movimiento dental ortodóncico pueden explicarse debido a que existen otros factores que influyen en los resultados que se puedan obtener, como son la densidad y altura ósea, el área radicular, la fuerza y función muscular, la interdigitación oclusal y el crecimiento esquelético. Estos factores deben tenerse en cuenta en futuros estudios.

\section{Fondos}

Esta investigación fue financiada por el CODI, Comité para el Desarrollo y la Investigación, Universidad de Antioquia, Medellín, Colombia (CODIGO: 006-2010) y recursos propios.

\section{Agradecimientos}

Al centro Radiológico Imágenes y Especialistas, por su ayuda en la toma de las imágenes tomográficas.

A todas aquellas personas que permitieron el desarrollo de este proyecto.

\section{Referencias}

1. McNamara JJ. Components of class II malocclusion in children 8-10 years of age. Angle Orthod. 1981; 51(3): 177-202. doi: 10.1043/0003-3219(1981)051<0177:COCIMI>2.0.CO;2.

2. Vasquez MJ, Baccetti T, Franchi L, McNamara JAJ. Dentofacial features of Class II malocclusion associated with maxillary skeletal protrusion: a longitudinal study at the circumpubertal growth period. Am J Orthod. Dentofacial Orthop. 2009; 135(5): 568.e1-7; discussion 568-9. doi: 10.1016/j.ajodo.2007.05.026.

3. Lim J-K, Jeon HJ, Kim JH. Molar distalization with a miniscrew-anchored sliding jig. J Clin Orthod. 2011; 45(7): 368-377.

4. Wallshein A. Design of an improved sliding jig. J Clin Orthod. 1974 ;8(8): 473.

5. Gianelly AA, Vaitas AS, Thomas WM, Berger DG. Distalization of molars with repelling magnets. J. Clin. Orthod. 1988; 22(1): 40-44. 
6. Lucato AS, Amelia S, Vedovello S. Sliding Jig: confecção e mecanismo de ação. Rev Clín Ortodon Dental Press. 2004: 10-17.

7. Limsiriwong S, Khemaleelakul W, Sirabanchongkran S, Pothacharoen P, Kongtawelert ., Ongchai S, et al. Biochemical and clinical comparisons of segmental maxillary posterior tooth distal movement between two different force magnitudes. Eur J Orthod. 2018; 40(5): 496-503. doi: 10.1093/ejo/cjx092.

8. Rodríguez E. Elasticos. In: Ortodoncia contemporanea. Diagnóstico y tratamiento. 3a ed. Medellín: AMOLCA; 2019.

9. Hixon EH, Aasen TO, Clark RA, Klosterman R, Miller SS, Odom WM. On force and tooth movement. Am J Orthod. 1970; 57(5): 476-478.

10. Kanchana P, Godfrey K. Calibration of force extension and force degradation characteristics of orthodontic latex elastics. Am J Orthod. Dentofacial Orthop. 2000; 118(3): 280-287. doi: $10.1067 / \bmod .2000 .104493$.

11. Philippe J. Mechanical analysis of Class II elastics. J Clin Orthod. 1995; 29(6): 367-372.

12. Baty DL, Storie DJ, von Fraunhofer JA. Synthetic elastomeric chains: a literature review. Am J Orthod. Dentofacial Orthop. 1994; 105(6): 536-542. doi: 10.1016/S0889-5406(94)70137-7.

13. Tweed C. Clinical Orthodontics. St Louis: Mosby; 1966.

14. Alves P, Bolognese AM, Gomes Souza MM. Movimiento distal de molares usando o SlidingJig. R Clin Orthon Dent Press. 4(6): 83-89. Disponible en: http://pesquisa.bvsalud.org/portal/ resource/es/lil-436377Accessed.

15. Burbano AE, Guerrero YE, Maria de los Reyes Al. Efectividad del gancho deslizante de protracción inferior para corregir clase II división 2. Univ Odontol. 2007; 26(58): 13-17.

16. Gianelly AA. Distal movement of the maxillary molars. Am J Orthod Dentofacial Orthop. 1998; 114(1): 66-72.

17. Chung KR, Kim SH, Choo H, Kook YA, Cope JB. Distalization of the mandibular dentition with mini-implants to correct a Class III malocclusion with a midline deviation. Am J Orthod. Dentofacial Orthop. 2010; 137(1): 135-146. doi: 10.1016/j.ajodo.2007.06.023. 
Comparación del movimiento del canino y molar superior utilizando elásticos de clase II con y sin Sliding Jig: un ensayo clínico aleatorizado

18. Aras I, Pasaoglu A. Class II subdivision treatment with the Forsus Fatigue Resistant Device vs intermaxillary elastics. Angle Orthod. 2017; 87(3): 371-376. doi: 10.2319/070216-518.1.

19. Cazenave C. Class II subdivision in adults: use of miniscrews anchorage in mixed technique. Orthod Fr. 2014; 85(4): 341-354. doi: 10.1051/orthodfr/2014022.

20. Scarfe WC, Azevedo B, Toghyani S, Farman AG. Cone Beam Computed Tomographic imaging in orthodontics. Aust Dent J. 2017; 62(Suppl 1): 33-50. doi: 10.1111/adj.12479.

21. Nasseh I, Al-Rawi W. Cone Beam Computed Tomography. Dent Clin North Am. 2018; 62(3): 361-391. doi: 10.1016/j.cden.2018.03.002.

22. Baumgaertel S, Palomo JM, Palomo L, Hans MG. Reliability and accuracy of cone-beam computed tomography dental measurements. Am J Orthod Dentofacial Orthop. 2009; 136(1): 18-19. doi: 10.1016/j.ajodo.2007.09.016.

23. Polat-Ozsoy O, Kircelli BH, Arman-Ozcirpici A, Pektas ZO, Uckan S. Pendulum appliances with 2 anchorage designs: conventional anchorage vs bone anchorage. Am J Orthod. Dentofacial Orthop. 2008; 133(3): 339.e9-339.e17. doi: 10.1016/j.ajodo.2007.10.002.

24. Patel MP, Janson G, Henriques JFC, de Almeida, RR, de Freitas MR, Pinzan A, et al. Comparative distalization effects of Jones jig and pendulum appliances. Am J Orthod Dentofacial Orthop. 2009; 135(3): 336-342. doi: 10.1016/j.ajodo.2007.01.035.

25. Anchorage R. Elastic FOR, Upper TB. U.S. Patent Jun 25, 1985;(19).

26. Quirós Alvarez O. Distalizacion de molares superiores sin auxilio de extraorales. Acta Odontológica Venez. 1993; 31:33-36.

27. Chiu PP, McNamara JAJ, Franchi L. A comparison of two intraoral molar distalization appliances: distal jet versus pendulum. Am J Orthod Dentofacial Orthop. 2005; 128(3): 353-365. doi: 10.1016/j.ajodo.2004.04.031.

28. Carano A, Testa M. The distal jet for upper molar distalization. J Clin Orthod. 1996; 30(7): 374-380.

29. Pattabiraman V, Kumari S, Sood R. Mini-implant-supported sliding jig. Orthodontics (Chic.). 2011; 12(4): 396-399. 